\title{
A Situated Learning Perspective on Learning Object Design
}

\author{
Roderick A. Farmer and Baden Hughes \\ Department of Computer Science and Software Engineering \\ The University of Melbourne \\ Parkville, VIC 3010, Australia \\ \{raf, badenh\}@cs.mu.oz.au
}

\begin{abstract}
Recent work suggests that learning object design can be improved by greater integration of instructional design, learning theory and software development methodologies. Despite this, there is a lack of research in the field that seeks to establish an association between the contextualised nature of learning object design and empirical properties of learner-computer interaction. In addressing this issue, we argue for a situated learning perspective on learning object design. Using the CASE framework as an exemplar of situated learning, we describe an holistic approach to eliciting socio-cultural properties of learning objects.
\end{abstract}

\section{Introduction}

It has been suggested that successful design of learning objects (LOs) necessitates incorporation of instructional design and learning theories with current LO design methodologies $[4,8]$. This requirement is symptomatic of many computer-mediated activities in which developers of learning systems are confronted with an emerging "socio-technical" gap, or the separation of tasks which are able to be supported technically from those which can be supported socially [1]. Current problems include the integration of learning theory and instructional design in LO creation; the lack of robust and holistic frameworks for LO design; and the ability of LO properties to account for computer-supported collaborative learning activities.

In response, we argue that there is a need to shift focus from the structural aspects of design towards holistic, socially-informed aspects for effective design of LOs. This paper applies one example of such a model, CASE [6], which can be used to describe the properties of a LO from the perspective of situated task analysis. We believe this perspective is informative, and provides new potential to realising learning objectives in the context of collaborative work, personalised and autonomous learning. Furthermore, the proposed model is expected to contribute to qualitatively greater learning outcomes and consequently a better return-on-investment [4].

We adopt the position that "learning object[s] should be that elemental constituent component that cannot be broken down any further and is ready for instructional use in different combinations" [2, p. 307]. By interpretation, then, LOs have a constrained scope which delineates them from general purpose software tools and establishes them as independent entities. A by-product of this quality is that an LO should be deployable across numerous domains. Wellconstructed LOs provide a domain-independent model of some aspect of learner-computer interaction, therefore affording a channel for increased stakeholder collaboration in transdisciplinary environments.

One consistently omitted topic of discussion is that of context, and its valency - what should be the role of context and situatedness in LO design? We must first recognise that knowledge and learning are embedded within sociocultural frames of reference [11]. Failure to include context in design and evaluation processes effectively ignores the issues and associated benefits of learner autonomy, as well as the embeddedness of collaborative activity within culturally-historic communities of practice. Rather than removing context from LO design, as promoted in [9], we embrace it, approaching LO description and evaluation from a learner-centred and situated perspective.

It has been proposed that for LOs to be inherently valuable, instructors, learners and software agents should be able to easily locate, aggregate and evaluate them [4]. Hence LOs need to be differentiated, described (in metadata) at the levels of content, concepts, skill impact, software, hardware and other usage requirements [4].

In order to combine and sequence LOs to achieve expected learning outcomes, object design must be commensurate with actual learning processes, an approach currently lacking in existing models. As a general discussion, Daniel 
and Mohan [4] propose a model for evaluating learning objects across four (largely technical) dimensions: content design, back-end delivery, front-end presentation, and the learning process. Contrastively, we have found it instructive to focus on content design and learning process evaluation. In addition, we believe that the two processes are not distinct phases, yet remain inextricably linked as learning materials implicitly represent cognitive and social interaction elements of an activity [5].

\section{A Situated Learning Perspective}

Situated learning is entrenched within the paradigms of situated cognition [3] and social learning theories [11]. According to [11], learning is seen as a process or function of activity within a community of practice. Central to situated learning perspectives, as present in $[7,5]$, is the importance of learning in the construction of subjectively relevant knowledge and individual learning skills. Whilst not dismissing the significant contributions from cognitive processing accounts of learning, situated learning perspectives posit the primacy of participation in authentic social interaction in order for learning to occur. Additionally, a situated learning approach to LO design goes beyond the structural and ontological representations of learning materials and learning outcomes [9], and demands the consideration of socio-technical factors in technology-mediated learning environments. A corollary of this premise is that LO design becomes inseparable from domain specific contexts and communities of use.

We argue that applying a situated learning approach to LO design is crucial for further advancement of the field as it recognises that understanding motivation, situated awareness, collaboration, conflict and negotiation, context, and learner-centredness are critical to developing effective learning environments $[6,3,5]$. Therefore, designing learning systems requires consideration of cognitive, sociocultural, organisational and environmental conditions of use. By providing a means to capture salient aspects of cognition and social interaction within a learning paradigm, the CASE framework (Cognition, Activity, Social Organisation, and Environment) [6] provides an integrative approach to the design of LOs as learning tools.

\section{The CASE framework}

The four components of CASE (Cognition, Activity, Social Organisation and Environment) each depict a level of investigation of situated learning. Likely influential factors impacting learning activities are situated within corresponding components of the model. Components are not mutually exclusive and may be used summatively or pro- ductively (depending on the desired analysis) to indicate learner-interface complexity at each level of abstraction.

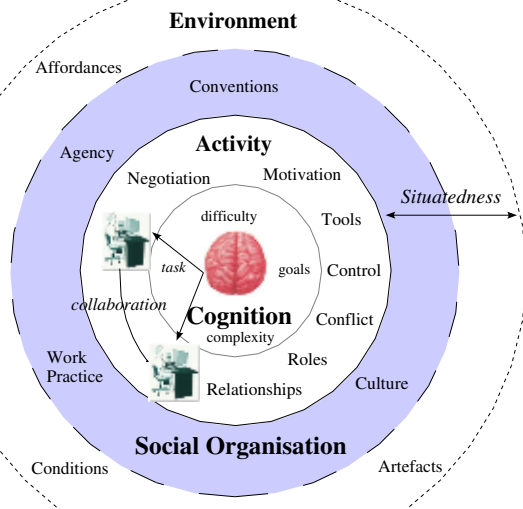

According to CASE, interaction can not be modelled linearly, as lower-level events like error handling and information incoherence may affect higher-level meta-cognitive fuctions, subsequently impacting motivation, planning, attention, and immediate task-orientation [5]. Task complexity, difficulty and contextual conditions may non-uniformly impact learner resource allocation according to a learner's level of expertise and available scaffolding within an activity $[10,3,11]$. As learning is a social process, and therefore typically ill-structured, dynamic and non-deterministic, incorporating the situatedness of an activity during design is likely to result in richer data reflecting a wider range of influential factors surrounding learning.

In CASE, Environment modelling is primarily concerned with questions of affordances, artefacts, and conditions. Affordances suggest naturally occuring relations between agents in the environment. Artefacts are those materials and work products that are used by the learning system (eg books, microphones, monitors). Modelling Social Organisation emphasises aspects of culture, conventions, and agency in communities of practice. Modifying the social organisation in which learning occurs is likely to not only affect agency, but also organisational work practice.

Activity is principally concerned with capturing toolmediated, subject-motivated interaction. Informed by Activity Theory [7, 5], we are interested in modelling the relationships and roles in agent (actors and artefacts) collaboration, how this polymotivates actions within the activity, and how in turn, conflicts and learner control strategies impact emerging cognitive tasks. The component of Cognition consists of three primary factors: task complexity (focuson-form, divided attention, information priming, associativity of the construct); conscious subject goals (motivation, task prioritisation), and task difficulty (reflecting transient qualities such as intelligence, aptitude, gender etc.). 
Whereas modelling goal structures and cognitive difficulty will assist in understanding ill-structured, emergent sociocollaborative practices, cognitive complexity is highly relevant to analysing learner-computer interaction in wellstructured, fixed scenarios.

\section{Discussion}

One of the benefits arising from the adoption of CASE for learning object description and evaluation is the ability to integrate instructional design and social theory considerations into learning object design through its foundations in situated learning. Other substantial benefits can be observed through adoption of this framework, especially in the area of Component-Based Software Development (CBSD). A central process within CBSD is that of component qualification. In order to build systems that depend largely upon the adaptation and aggregation of reusable components, such as LOs, we must first be able to elicit and describe the functional properties of an object, as well as determine the overall utility of selecting and using an object. This requires solving various socio-technical issues such as organisational and social constraints which can be described as evaluative criteria for object selection. Moreover, re-use strategies in CBSD contexts often lack sufficient consideration of end-user constraints, including various sociopsychological constraints. Addressing the paucity of frameworks that adequately monitor and address these concerns will increase the "fit-for-purposefulness" (and likely returnon-investment) of component-based tools.

There are various assumptions within the CASE framework which require further consideration. Notably, the framework currently assumes that formal properties and associated ontologies exist that can be accessed as meta-data of the LO; and pedagogical drivers of learning systems development will be equally attentive to evaluation as to implementation, which is not always the case. In addition, the framework is presently unable to adequately describe task sequencing criteria which requires an evaluation of complexity, causality (between sequences) and various other pedagogical needs [10]. It is possible that longitudinal empirical investigations across several domains and situated activities may uncover causal relationships between the components of the CASE model, such as increased cognitive complexity of an activity in large actor networks. This represents a significant interest within our current research activities.

\section{Future Work and Conclusions}

Prescribed frameworks are inherently static and therefore will tend to fail to capture all modes and aspects of interaction. The ability of the CASE framework to model individual and collaborative aspects of cognition, interaction and sociocultural constraints renders it an attractive tool for LO design and evaluation. Whilst the CASE framework is capable of modelling the spatio-temporal conditions of learning and interaction, further research is required for evaluating the impact of dynamic conditions during collaboration. The benefit of this activity, as identified in [11,5], is the ability to tie broader historical trends to present (and future) activities. Research remains to be undertaken regarding the development of ecologically-relevant ontological models for indexing learning material within a socioculturally relevant domain of use [8]. We are currently investigating these problems with an intent to provide a tractable means of imbuing LOs with CASE properties.

\section{References}

[1] M. S. Ackerman. The intellectual challenge of cscw: The gap between social requirements and technical feasibility. Human-Computer Interaction, 15(2-3):179-203, 2000.

[2] N. Boskic. Learning objects design: What do educators think about the quality and reusability of learning objects. In Proceedings of the 3rd IEEE International Conference on Advanced Learning Technology (ICALT'03), pages 306-307. IEEE Computer Society Press, 2003.

[3] J. S. Brown, A. Collins, and P. Duguid. Situated cognition and the culture of learning. Educational Researchers, 18(1):32-42, 1989.

[4] B. K. Daniel and P. Mohan. A model for evaluating learning objects. In Proceedings of the IEEE International Conference on Advanced Learning Technologies (ICALT'04), pages 56-60. IEEE Computer Society Press, 2004.

[5] Y. Engeström. Activity theory and individual and social transformation. In Y. Engeström, R. Miettinen, and R. Punamaki, editors, Perspectives on Activity Theory. Cambridge University Press, New York, 1999.

[6] R. A. Farmer. Situated task analysis in learner-centred call. In P. Zaphiris, editor, User-Centred Computer Aided Language Learning. Idea Group Publishing, Hershey, PA, 2006. In Press.

[7] A. N. Leont'ev. Activity, consciousness and personality. Prentice Hall, Englewood Cliffs, NJ, 1978.

[8] T. Nabeth, A. A. Angehrn, and R. Balakrishnan. Integrating 'context' in e-learning systems design. In Proceedings of the IEEE International Conference on Advanced Learning Technologies (ICALT'04), pages 355-359. IEEE Computer Society Press, 2004.

[9] P. E. Parrish. The trouble with learning objects. Educational Technology, Research and Development, 52(1):49-67, 2004.

[10] P. Robinson. Task complexity, cognitive resources and second language syllabus design: A triadic theory of task influences on sla. In P. Robinson, editor, Cognition and Second Language Instruction, pages 285-317. Cambridge University Press, Cambridge, 2001.

[11] L. S. Vygotsky. Thought and language. MIT Press, Cambridge, MA, 1986. Translation by Alex Kozulin. 


\section{University Library}

\section{- M M I N E R VA A gateway to Melbourne's research publications}

Minerva Access is the Institutional Repository of The University of Melbourne

Author/s:

Farmer, R. A.;Hughes, B.

Title:

A Situated Learning Perspective on Learning Object Design

Date:

2005

Citation:

Farmer, Roderick and Hughes, Baden (2005) A Situated Learning Perspective on Learning Object Design, in Proceedings, of 5th IEEE International Conference on Advanced Learning Technologies (ICALT 2005), Kaohsiung.

Publication Status:

Published

Persistent Link:

http://hdl.handle.net/11343/34047 